\title{
Essais
}

ESSAIS

Revue interdisciplinaire d'Humanités

$17 \mid 2021$

Quels lieux pour les morts?

\section{Les pratiques funéraires contemporaines japonaises : quels lieux pour les morts et pour la mort au Japon?}

Contemporary Japanese Funeral Practices: What Places for the Dead and for Death in Japan?

Fabienne Duteil-Ogata

\section{(2) OpenEdition}

Journals

Édition électronique

URL : https://journals.openedition.org/essais/8635

DOI : $10.4000 /$ essais. 8635

ISSN : 2276-0970

Éditeur

École doctorale Montaigne Humanités

Édition imprimée

Date de publication : 15 avril 2021

Pagination : 75-86

ISBN : 979-10-97024-09-3

ISSN : $2417-4211$

Référence électronique

Fabienne Duteil-Ogata, « Les pratiques funéraires contemporaines japonaises : quels lieux pour les morts et pour la mort au Japon? », Essais [En ligne], 17 | 2021, mis en ligne le 27 avril 2021, consulté le 18 janvier 2023. URL : http://journals.openedition.org/essais/8635; DOI : https://doi.org/10.4000/ essais.8635 


\section{Les pratiques funéraires contemporaines japonaises : quels lieux pour les morts et pour la mort au Japon?}

\section{Fabienne Duteil-Ogata}

Afin de formuler quelques réponses à cette question, je présenterai tout d'abord le contexte général des pratiques funéraires traditionnelles centrées sur le culte des ancêtres dont l'ancrage historique et institutionnel s'appuie sur le bouddhisme depuis la période d'Edo (1603-1868) et qui accorde deux lieux privilégiés pour les morts : la tombe située dans le monastère bouddhique paroissial et l'autel des ancêtres disposé dans l'espace domestique de l'héritier en charge des cultes. J'analyserai, ensuite à partir de terrains ethnographiques, réalisés à Tôkyô, entre 2006 et 2012, dans le cadre du programme ANR Funérasie, comment de nouvelles pratiques semblent annihiler ce culte et la notion même d'ancêtre et promouvoir d'autres lieux pour les morts. Je conclurai en m'interrogeant sur le sens à donner à ces nouveaux lieux qui sont à l'origine de nouvelles représentations des morts et de la mort au Japon, créant une nouvelle ontologie.

\section{Contexte général des pratiques funéraires traditionnelles centrées sur le culte des ancêtres : la tombe paroissiale bouddhique et l'autel domestique bouddhique}

Le culte des ancêtres repose sur la filiation patrilinéaire créant une lignée au sein du groupe domestique, appelé en japonais ie, la maison ${ }^{1}$. Il s'effectue en ligne patrilinéaire et est traditionnellement pris en charge par le fils aîné (à la mort de ses parents) ; le fils cadet forme, quant à lui, une nouvelle lignée ancestrale. S'il n'y a pas de descendant masculin, on procède alors à l'adoption d'un gendre. La femme, quant à elle, appartient à la lignée de son mari.

Officiellement, selon la loi, le culte des ancêtres reste libre et relève du domaine de la liberté religieuse (art. 20 de la constitution), même si l'article 897 du code civil de 1948 précise que : "la propriété des documents

1 Laurence Caillet, La maison Yamazaki, Paris, Plon, 1991, p. 405. 
généalogiques de la famille, les objets de culte et la tombe sont donnés à l'un des héritiers qui préside le culte des ancêtres $"^{2}$. Le code civil laisse bien sûr le choix de l'héritier (homme ou femme). Toutefois le poids de la tradition persiste et de nos jours encore c'est souvent le fils aîné qui prend en charge le culte des ancêtres de sa famille, une fois le père et la mère décédés.

Rappelons tout d'abord que la religion japonaise traditionnelle est de nature syncrétique. Elle a assimilé, au fil du temps, deux principaux courants religieux institutionnels : le shintô et le bouddhisme. Le shintô est une religion animiste et polythéiste. Les hommes, divinités, la nature ou les objets sont de nature identique car animés des mêmes tama (forces vitales), créant ainsi une continuité ontologique. Le shinto insiste sur l'immanence du monde et se concentre plutôt sur la vie terrestre même si selon la pensée eschatologique, le mort devient ancêtre, puis divinité tutélaire.

Le bouddhisme, quant à lui, offre une pensée eschatologique plus développée, un "salut-délivrance " individualisé et prend en charge les pratiques funéraires délaissées par le shintô qui considère la mort comme un état de pollution extrême, antinomique avec la pureté des divinités shintô. Le processus d'ancestralisation relève donc de la compétence du bouddhisme ; cependant, à l'issue d'un cycle rituel de trente-trois ans ou cinquante ans, si les rites bouddhiques ont été effectués selon les règles, l'ancêtre peut intégrer le panthéon shintô et devenir ancêtre protecteur de la communauté dont il est issu ; il viendra alors protéger et exaucer les vœux des hommes.

Les raisons de la monopolisation de l'espace funéraire par la sphère bouddhique sont principalement d'ordre politique et historique. Si dès l'arrivée du bouddhisme sur le territoire japonais au $\mathrm{VI}^{\mathrm{e}}$ siècle, la cour adopta les pratiques funéraires du bouddhisme, ce n'est qu'au XVII' siècle qu'une partie plus importante de la population, mais encore minoritaire, eut accès aux pratiques funéraires bouddhiques. C'est en effet dans le cadre de la politique du Shôgunât des Tokugawa à l'époque d'Edo (1603-1868) que les pratiques funéraires bouddhiques se développèrent au sein de la population sous l'effet de plusieurs ordonnances $^{3}$ qui demandaient à la population de s'inscrire à un monastère bouddhique et d'effectuer les rites bouddhiques, notamment les rites funéraires.

La première ordonnance de 1638 imposa à la population de s'inscrire à un monastère bouddhique local et d'obtenir " un certificat d'appartenance à l'institution bouddhique locale " comme preuve de leur non-appartenance

2 Patrick Beillevaire, «La famille instrument et modèle de la nation japonaise », in André Burguière, Christiane Klapisch-Zuber, Martine Ségalen et Françoise Zonabed, Histoire de la famille, Tome 3, Le choc des modernités, Paris, Armand Collin, 1986, p. 322.

3 Fumio Tamamuro, "Jidan : idéologie des rapports qui liaient monastères bouddhiques et familles paroissiales à l'époque d'Edo ", in Cahiers d'études et de documents sur les religions $d u$ Japon, Paris, Alpha bleue, 1997, p. 87-110. 
chrétienne. En effet, après les premières missions des Jésuites portugais en 1549, le christianisme fut interdit au Japon en 1614 (pour des raisons politiques notamment dues à la peur d'une invasion et à la possible émergence de troubles au sein de l'empire récemment unifié). L'ordonnance de 1671 demandait à la population de déclarer au monastère les décès et d'effectuer des funérailles bouddhiques ; il fut alors possible, à cette époque, pour une infime tranche de la population, d'ériger une pierre tombale. L'ordonnance de 1700 imposait aux personnes affiliées aux monastères bouddhiques de leur localité de procéder aux rituels commémoratifs du jour anniversaire de la mort du défunt ainsi qu'aux fêtes calendaires bouddhistes, et si l'un des membres venait à négliger ces rites, il n'obtenait pas de certificat, il était alors soupçonné d'être chrétien et encourait la peine capitale. Ces obligations administratives eurent pour corollaire l'établissement du système des danka " paroissiens du monastère " rassemblant des groupes domestiques (ie " la maison ") qui sont affiliés à un monastère bouddhique (de génération en génération) et qui participent financièrement à la vie du monastère par des dons.

Ainsi, le mort devient un ancêtre individualisé par un processus de rites ancestraux qui durent généralement 33 ans ou 50 selon les écoles de pensée bouddhique ; le défunt prend alors un nom posthume bouddhique et devient un ancêtre protecteur de la lignée familiale. Les différents rites bouddhiques permettant la mise en ouvre de ce processus sont assurés par les moines des monastères bouddhiques. Les rites funéraires ont donc lieu dans deux espaces différents : l'espace de la tombe située dans l'enceinte du monastère où se trouve le corps du défunt (urne = cendres + os) et l'autel domestique bouddhique installé dans la résidence de l'héritier de la famille en charge du culte, dans lequel est enchâssé le défunt, matérialisée par la plaquette funéraire et le portrait photographique funéraire. Les rites post-mortem sont donc les garants de la bonne marche du processus d'ancestralisation. Les visites sur la tombe constituent avec les rites à l'autel domestique bouddhique des ancêtres les pratiques funéraires les plus importantes.

\section{De nouvelles pratiques funéraires encore minoritaires qui semblent annihiler le culte des ancêtres et créer de nouveaux lieux pour les morts}

À partir de terrains ethnographiques, réalisés à Tôkyô, entre 2006 et 2012, dans le cadre du programme ANR Funérasie ${ }^{4}$, je présenterai plusieurs nouvelles pratiques qui semblent annihiler ce culte et être à l'émergence de

4 Ce programme de recherche ANR FunerAsie intitulé « l'expansion de l'industrie funéraire en Asie du Nord-Est (Chine, Corée, Japon) enjeux économiques, spatiaux et religieux » a abouti à la publication de deux volumes : Natacha Aveline-Dubach, La place des morts dans les mégalopoles d'Asie orientale, Paris, 2013, et Natacha Aveline-Dubach, Invisible population, Lanham, Lexington Books, 2012. 
nouveaux lieux pour les morts: les «tombes ou ossuaires collectifs aux cultes éternels "; le lancer des cendres et les objets funéraires à portée de main.

Depuis les années 1990 et l'éclatement de la bulle foncière, de nouvelles pratiques funéraires ont vu le jour qui ne visent plus à célébrer le défunt pour qu'il devienne ancêtre protecteur de la famille. Ce qui est en jeu dans ces nouvelles pratiques c'est l'individualité du défunt, la mémoire du mort et non plus sa profondeur généalogique.

De nombreuses raisons concourent à cet état de fait. Le coût trop élevé des rites et leur longue durée (33 ans ou 50 ans) incitent de nombreux Japonais à choisir d'autres formes de sépulture pour ne pas faire peser sur leurs enfants le coût de l'enterrement, de l'entretien de la tombe au monastère bouddhique et des nombreux rituels. Liés à cet aspect économique, l'aspect social, la remise en cause de l'institution du ie (la " maison ») basée sur la filiation patrilinéaire privilégiant le fils aîné, perdent leur force dans une société contemporaine urbanisée et plus individualisée où la famille nucléaire est centrale. Aussi, la croyance dans la notion d'ancêtre de génération en génération est-elle aussi peu à peu remise en cause au profit de la seule mémoire du défunt individualisé, ce qui concourt à l'avènement de nouveaux espaces funéraires.

\section{Les tombes ou ossuaires collectifs aux cultes éternels}

Ces tombes se distinguent des tombes paroissiales bouddhiques du groupe domestique sur deux points : les individus réunis dans ces tombes n’appartiennent pas au même groupe domestique, et la durée de leur culte est éternelle. Généralement les monastères bouddhiques peuvent assurer un culte éternel du fait de la résidence du moine dans le monastère même. La première tombe collective " aux cultes éternels " a été créée en 1985. Puis, dans les années 1990, de nombreux monastères bouddhiques et cimetières municipaux ont proposé ce service. En 2009, on dénombrait près de 1000 tombes collectives aux cultes éternels sur l'ensemble du territoire nippon alors qu'elles n'étaient que 400 en 2006. Construite en 1989 à l'initiative de la femme du bonze du monastère Jôkôji à Tôkyô, cette tombe collective répond à la demande de certains paroissiens du monastère : les femmes (célibataires, divorcées) ou celles qui ne désirent pas reposer dans la même tombe que leur mari. L'idée de laisser la liberté à l'individu de choisir le lieu de sa sépulture a également été retenue par le monastère Tôchôji en créant une " tombe individuelle du vivant " Seizenkôjinbô gérée par "l'association du Lien " (en no kai). En 2009, cette association comptait 8500 membres, dont deux tiers étaient des femmes. Seuls $20 \%$ étaient décédés. La majorité des adhérents $(78 \%)$ résident à Tôkyô ou dans les préfectures limitrophes, et $70 \%$ d'entre eux s'inscrivent en couple. Leur principal motif d'adhésion est l'absence de descendant ou, dans une moindre mesure, le fait d'avoir une fille qui ne pourra pas poursuivre le culte des ancêtres (même si légalement c'est possible). La gêne d'imposer l'entretien 
de la sépulture à leurs enfants est également une motivation. Parmi les autres adhérents, $18 \%$ sont des personnes seules et les $12 \%$ restants constituent des " couples inédits ": des frères et sœurs, ou une mère et son enfant. Notons que ce sont les liens affectifs au sein de la famille qui déterminent ces paires plutôt que la filiation patrilinéaire.

La tombe collective aux cultes éternels a aussi ses avatars virtuels. En mars 2006, le bonze du monastère Kudokuji à Tôkyô a lancé " la tombe ordinateur " puis la " tombe internet ». Pour une somme de 150000 à 250000 yens (1 400 à 2300 euros), on peut créer sa tombe virtuelle (avec des textes, photographies, vidéos) et bénéficier on non d'un culte éternel avec rites mensuels. Les cendres sont quant à elles disposées dans une tombe collective. Le souscripteur désigne en outre sa propre " filiation ", car il choisit de son vivant les personnes qui viendront visiter sa tombe après sa mort, en leur communiquant un identifiant (ou une carte) et un code d'accès pour visualiser sa page web. Il créé une communauté d'internautes, une "nouvelle parenté », une filiation choisies.

La nouvelle forme de tombe "with pet ", associant l'urne d'une personne et celle de son animal de compagnie, a été créée en 2003 par l'entreprise de pompes funèbres Ohnoya intervenant dans le cimetière Izumi Joen. Loin d'être anecdotique, ce marché est en constante expansion. Depuis 2003, l'entreprise Ohnoya a produit 2000 tombes de ce type. La demande émane surtout de personnes âgées désirant reposer à côté de leur seul être cher. Dans ce même cimetière Izumi Joen se trouve une autre formule innovante qui rencontre un grand succès : " les tombes sous les arbres " jumokusô. Ce dispositif de sépulture a été introduit pour la première fois au Japon en 1999 dans le monastère bouddhique Shôunji (préfecture d'Iwate). L'objectif était de préserver l'environnement. En plantant un arbre au-dessus de l'urne du défunt, non seulement on réduisait l'espace alloué au mort mais on évitait d'utiliser de la pierre, tout en donnant naissance à une nouvelle forêt. La sociologue Haruyô Inoue a créé une association sans but lucratif NPO (Non Profit Organization) "Ending Center " et adapté ce concept rural à une demande urbaine avec " la tombe sous les cerisiers ", sakurasô bochi. Cette réalisation s'inspire en partie du monastère Shôunji, mais également du cimetière suédois du quartier Lidingö de Stockholm. La première "tombe sous les cerisiers ", créée en avril 2005, comprend une parcelle collective (100 concessions) et 250 lots individuels pouvant contenir chacun une à cinq personnes, tous situés au-dessous de deux cerisiers. En un an et demi, toutes les concessions individuelles ont été vendues (400 000 yens). En mai 2007 a été lancée une nouvelle tombe collective sous les arbres, composée de 300 parcelles individuelles avec, au centre, l'arbre Soyogo (ilex pedunculosa) à

5 Fabienne Duteil-Ogata, "Nouvelles pratiques funéraires japonaises : de la tombe ordinateur à la tombe-online ", in Fabienne Duteil-Ogata, Isabelle Jonveaux, Liliane Kuczynski et Sophie Nizard, Le religieux sur Internet, Paris, L'Harmattan, 2015, p. 231-246. 
feuilles persistantes. Haut de 5 à 10 mètres, cet arbre trône au milieu des emplacements qu'il protège de ses branches et atteint le ciel. Le succès de ces tombes a encouragé l'aménagement d'un troisième site en 2008, ciblant davantage des familles avec des concessions pour cinq personnes. Les parents achètent une concession pour eux et laissent la liberté à leurs enfants de les rejoindre s'ils en ont envie. Selon Haruyo Inoue ${ }^{6}$, le concept de "tombe sous les cerisiers " répond à quatre tendances sociétales : le manque d'héritier, l'individualisme, le double attachement des femmes à leur famille d'origine et leur famille par alliance, et la tendance au retour à la nature. La seconde nouvelle pratique funéraire met également en scène la nature.

\section{Le lancer des cendres dans la nature}

Le 15 octobre 1991, le journaliste Yasuda Mutsuhiko, créateur de "l'association pour la promotion de la liberté des funérailles ", (sôsô no jiỳu wo susumeru kai, Grave free promotion society), obtient des deux ministères compétents la légalisation de la pratique du lancer des cendres dans la nature. La nature (mer, montagne, espace, rivière) devient alors un lieu de sépulture. Les cendres et os réduits en poudre y sont dispersés sans qu'il y ait une possibilité d'identifier un lieu exact. Le lancer des cendres n'assigne a priori au mort aucun espace fixe, aucune trace, aucun rituel.

Déterritorialisé, dématérialisé, le corps mort se fond alors dans l'environnement naturel et redevient un élément naturel. Le retour du corps à la « mère nature " rappelle la consubstantialité de l'homme avec la nature, présente notamment dans l'animisme du shintô. Forte de 12000 membres, cette association présente dans tout le Japon, organise des événements (meetings, séminaires, colloques) et publie de nombreux ouvrages ${ }^{7}$. Elle procède à des lancers de cendres. De 1991 à 2009, elle avait effectué 1420 lancers pour 2469 personnes, soit $80 \%$ dans la mer, $18 \%$ dans la montagne et $2 \%$ en rivière ou dans les airs. Les prix des lancers varient selon la nature de la cérémonie (individuelle ou collective) et selon le lieu (mer, montagne, air, rivière). Ces montants restent toutefois bon marché au regard des prix des sépultures traditionnelles, de 120000 à 240000 yens (soit de 1000 à 2000 euros).

Les lancers de cendres collectifs dans la mer s'effectuent à bord de bateaux de taille moyenne pouvant accueillir une trentaine de personnes. Les cendres sont dispersées tour à tour dans la mer par chaque famille. Un à un, les membres de la famille lancent leurs petits sachets de cendres enveloppées dans une feuille

6 Haruyo Inoue, haka to kazoku no henyô [Les transformations de la famille et des sépultures], Tôkyô, Iwanami Shoten, 2003.

7 Mutsuhiko Yasuda \& Ikuo Nakamura, Shizensô to seikai no shîkyô [Le lancer des cendres dans la nature et les religions du monde], Tôkyô, Gaifusha, 2008. Mutsuhiko Yasuda, O haka ga nai to shinemasen [Quand on n'a pas de tombe alors on ne peut pas mourir ?], Tôkyô, Iwanami Shoten, 2002. 
blanche biodégradable ainsi que les pétales de roses, de chrysanthèmes et d'œillets qu'ils ont préparés dans un petit sac. Certains lancent également la boisson préférée du défunt et prononcent quelques paroles d'adieu; une fois que le sac est immergé, ils s'en retournent à leur place. Puis, les familles viennent chercher en mains propres le certificat attestant officiellement du lancer des cendres ainsi que la carte GPS indiquant l'endroit précis du lancer. Le certificat et la carte GPS sont les seules traces tangibles attestant de la cérémonie.

Madame A., professeur de médecine retraitée, explique que son mari, écrivain, a découvert l'association grâce à un article de presse et qu'il est devenu membre en 1993. Elle, n'a rejoint l'association qu'à la mort de son mari, en juillet 2008. "C'était une personne profondément libre qui n'avait aucun intérêt pour la religion, il voulait retourner à la terre, sans aucune cérémonie. Il aurait aimé que ses cendres soient dispersées sur le Mont Takao (Tôkyô), mais l'association n'effectue pas de lancer sur ce site, alors il a accepté que l'on fasse le lancer dans la mer car il a vécu son enfance en bord de mer. [...] Moi, personnellement, je n'avais rien prévu pour ma sépulture, en revanche, je ne voulais pas être dans la tombe de la famille de mon mari, mais depuis que j'ai fait le lancer des cendres, je pense que je demanderai à mon fils de le faire ainsi. [...] Le frère aîné de mon mari m’a demandé qu'une partie des cendres soit déposée dans la tombe familiale du monastère bouddhique. J'ai accepté, car vers la fin de sa vie, mon mari manifestait un sentiment d'attachement envers son "pays natal" (furusato). Toutefois, je n'effectuerai aucun culte là-bas. J'ai juste posé sa photo sur l'étagère. Je me sens maintenant apaisée, mon mari repose au fond de l'océan, selon ses vœux. "

Madame A. n'a pas de sentiment religieux même si elle a accepté de donner la moitié des cendres de son époux pour qu'il repose dans la tombe familiale. Chez elle, aucune trace de culte, seule la photo de son mari marque sa présence. Comme le mari de Madame A., celui de Madame Y. a découvert l'association par un article de presse de Yasuda. Fils unique, sans descendant et ne pratiquant aucune religion, il voulait lui aussi naturellement retourner à la nature. "Pendant les 49 jours qui suivirent les funérailles, j'ai gardé ses cendres à la maison, puis j'ai demandé à ce qu'elles soient réduites en poudre pour pouvoir les lancer dans l'océan. J'ai ensuite réparti les cendres en trois paquets, une partie pour la cérémonie dans la baie de Tôkyô près d'un de nos anciens domiciles, une autre destinée à Amalfi (Italie) car cet endroit nous a marqué et le reste est disposé ici sur l'étagère sur laquelle se trouve également le certificat du lancer et la carte GPS ».

Difficile de connaître exactement la population concernée par le lancer des cendres; il s'agirait toutefois de personnes qui ont des convictions écologiques voire animistes et qui refusent les pratiques des institutions religieuses.

C'est également ce qu'indique Monsieur Kitada, directeur de la société anonyme Kaze (vent) qui a procédé à plus de 700 lancers depuis 1998 : «Plus que des romantiques, on a affaire à des personnes de principes, des personnes 
qui ne veulent pas procéder aux funérailles, ni avoir de nom posthume bouddhique, ni lecture de sutra, ni tombe. Récemment la classe moyenne des salariés, sans descendance, divorcés ou célibataires choisit également ce mode de sépulture du fait de son coût peu élevé ». Le lancer des cendres commencerait donc à se démocratiser.

Le mort, sous forme de sachet de cendres, devient un artefact que l'on peut conserver chez soi ; ses cendres le matérialisent, le représentent, et le sachet de cendres devient ainsi un réel objet de culte. Le témoignage de Monsieur Kitada corrobore notre analyse : « 80 \% des personnes souscrivant au lancer des cendres désirent qu'une partie des cendres soit gardée dans l'espace domestique, pour servir d'objet de culte ; ceux qui lancent la totalité des cendres $(20 \%)$ le regrettent, mentionne-t-il ». Aussi, il est fréquent, que les sociétés anonymes spécialisées dans le lancer des cendres dans l'Océan Pacifique comme celle de Monsieur Kitada, proposent à leur clientèle des " croisières du souvenir ", memorial cruise sur le lieu du lancer des cendres afin que la famille se recueille une nouvelle fois pour effectuer un mémorial en lançant des fleurs ou pour procéder à un rituel religieux en demandant à un bonze d'effectuer un rite commémoratif, comme si l'absence de la matérialité du mort dans un lieu fixe était difficile à accepter, car les personnes se retrouvent sans objet physique déterminé, sans destinataire matérialisé, sans référent, pour effectuer un culte.

\section{Les objets funéraires à portée de main}

Les " objets funéraires à portée de main " (temoto kuyôhin) répondent à cette absence matérielle du mort. Il s'agit de micro-contenants de cendres (accessoires, pendentifs) ou de petits objets (plaques, diamants) fabriqués à partir des cendres des défunts, commercialisés par une association à but non lucratif et quelques entreprises privées ${ }^{8}$.

Monsieur N. a créé son entreprise en 1999, mais l'idée lui est venue en 1993, au décès de sa belle-mère. Il a trouvé que les funérailles ne servaient à rien car les rituels étaient incompréhensibles et réunissaient des personnes inconnues (famille éloignée, voisins du défunt). C'est alors qu'il a pensé à sa propre mort. Pour éviter la construction d'une tombe (dommageable pour l'environnement), il a tout d'abord envisagé le lancer des cendres, mais comme il voulait laisser une trace à ses enfants, il trouva une troisième solution : " la plaquette éternelle ». " Mon idée était que l'on puisse toucher la personne (ses cendres), la prendre dans ses mains, que l'objet ait une forme simple, sobre,

8 Jôji Yamasaki, O haka no shinpai muyô temoto kuyô no susume shinemasen [Inutile de s'inquiéter pour la tombe, recommandations pour des cultes funéraires à portée de mains], Tôkyô, Shôdensha, 2007. 
(pas comme les objets bouddhistes très décorés) ». Ces petites plaquettes de couleur pastel (ivoire, blanc, bleu, vert, marron) sur lesquelles sont gravés au laser le nom du défunt, ses dates de naissance et de mort ainsi qu'une photographie (en option) sont insérées dans un écrin que l'on peut disposer sur une table. Monsieur N. propose également des "Eternal Powder ", sortes de sphères transparentes dans lesquelles sont disposées les cendres sous forme de poudre blanche, pour qu'elles soient visibles.

Depuis 2000, existe le pendentif éternel, "eternal pendant ", plaquette cinéraire plus petite, encadrée de platine, d'argent ou d'or et portable autour d'une chaine. "Ce sont les femmes qui m’ont donné l'idée de créer ces pendentifs, elles voulaient que les plaquettes soient un bijou, à $80 \%$ ce sont les femmes qui achètent ces objets ». Monsieur N., a également créé la même gamme d'objets pour les animaux de compagnie (Eternal plate, Eternal Pendant, et Eternal Powder) au même prix. Majoritairement féminine, sa clientèle n'a pas de tombe (lancer de cendres) pour $70 \%$ d'entre elles, ou bien la tombe est trop éloignée du domicile pour les $30 \%$ restants ; ainsi en ayant un objet funéraire à portée de main, les personnes gardent un lien tactile avec la personne aimée ou l'animal disparu.

Madame T., responsable de la société LifeGem Japon, créée en 2005, m’explique également que sa clientèle est à $80 \%$ féminine. Le procédé a été mis au point en 2001 par l'américain Greg Herro créateur de la société LifeGem à Chicago (Illinois). Madame T. explique qu'il suffit de 50 grammes de cendres pour confectionner 1 diamant. À $60 \%$, ils sont montés en bague et à $40 \%$ en pendentifs. Selon la taille du diamant (de 0,2 carat à 1,5 carat) et la couleur, les prix varient de 398000 yens à 2698000 yens. Il faut évidemment ajouter au diamant le prix du bijou lui-même (bague, pendentif et chaîne ou boucle d'oreille) et l'inscription laser sur le diamant qui est optionnelle (nom du défunt, date de naissance et de mort, épitaphe). "Le diamant est un souvenir unique, indestructible, éternel, beau, que l'on peut voir et toucher " dit-elle.

D'autres entreprises proposent des objets funéraires à portée de main, sous forme d'urnes sculptées dans la pierre ou en céramique en forme d'œuf, de moine bouddhiste, de pyramide, de lune, de porte-bonheur, de rosaire, de flamme, des pendentifs en forme de cœur, d'étoile, de croix, de porte-photo.

Tous ces objets ont en commun de mettre en avant l'esthétique, d'impliquer un rapport sensible (visuel et tactile) et individualisé avec l'être disparu, qui, le plus souvent, ne passe pas par un rituel religieux et réduit drastiquement l'espace funéraire à l'urne cinéraire, à un bijou, ou un artefact portable sur soi ou mobile. 
Nouveaux lieux: nouvelles représentations des morts et nouvelle ontologie de la mort au Japon?

Ces nouveaux dispositifs mettent en avant l'individu et non plus la famille traditionnelle ni même la famille conjugale : les règles de filiation sont supplantées par les rapports affectifs. Le choix des partenaires dans l'au-delà et des successeurs remet en cause la perpétuation de la lignée ancestrale patrilinéaire, et le culte des ancêtres et élargit l'espace social des défunts; il ne s'agit plus de retrouver dans le même cavurne les descendants d'une même famille en ligne patrilinéaire mais d'ouvrir cet espace funéraire à d'autres personnes sans lien de parenté, des personnes qui ont souscrit au même dispositif funéraire, à la même tombe collective, allant même jusqu'à ouvrir cet espace aux animaux de compagnie.

Ces nouveaux dispositifs funéraires créent également de nouveaux espaces dédiés aux morts (sépulture et culte). Ainsi dans le cas des tombes collectives aux cultes éternels, le bonze devient la seule personne légitime pour pacifier le mort et effectuer les cultes éternels. L'espace dévolu aux morts (aux cendres) et au culte du mort se trouve ainsi concentré dans une seule tombe, quelle que soit la configuration choisie (sous les arbres, tombe-ordinateur, tombe avec animal de compagnie, etc.).

La deuxième forme de pratiques funéraires à savoir le lancer des cendres dans la nature semble réduire davantage encore l'espace dévolu à la sépulture et au culte du mort, comme si cette pratique avait le pouvoir d'annihiler toute trace matérielle du défunt, les cendres ayant pour fin de se dissoudre dans les éléments naturels ; seul perdure à minima un culte domestique mémoriel incarné par la représentation photographique du défunt rappelant son souvenir et la carte GPS indiquant le lieu du lancer.

Les objets funéraires à portée de main participent également de cette réduction drastique de l'espace dédié aux morts. Ils sont souvent le fait des femmes qui tiennent à garder une proximité physique, tactile, avec les seules cendres qui n’ont pas été lancées. L'objet funéraire à portée de main est alors assimilé à un talisman ou une relique sans qu'aucun rituel ne lui ait octroyé ce statut.

De manière générale, l'espace destiné à la sépulture du mort s'en trouve réduit, concentré voire annihilé, presque totalement. Les considérations environnementales pour limiter le déboisement des montagnes (lieu privilégié des morts) et restreindre l'usage des pierres tombales sont, semble-t-il, une préoccupation des nouveaux acquéreurs de ces nouveaux dispositifs funéraires. Ces nouvelles pratiques désavouent le culte des ancêtres privilégiant un culte mémoriel. Perdure toutefois l'espace alloué à la célébration de la mémoire du mort. Cet espace se trouve lui aussi le plus souvent réduit à sa plus simple expression : l'autel domestique bouddhique a laissé place à une interface informatique, à une photo ou à un objet. Constatons, par ailleurs, que ces nouveaux 
espaces de culte mémoriel sont plus mobiles qu'avant, notamment pour les objets cinéraires et les tombes virtuelles permettant ainsi aux vivants d'être, à tout instant, n’importe où, en coprésence virtuelle ou physique avec les morts.

Le mort n'est plus un ancêtre, mais un être (qui demande à être pacifié pour les adeptes des tombes collectives) et dont le culte mémoriel peut toutefois protéger ou rassurer les vivants, comme un talisman ou une relique peut le faire, par la proximité de sa présence (objet, photo), ou bien encore redevenir un simple élément de la mère nature (lancer des cendres). N'assiste-t-on pas à un changement ontologique du mort ? L'ancêtre issu d'une filiation patrilinéaire a laissé place à un défunt dont les liens reposent sur des relations affectives, comme si le mort en perdant sa profondeur généalogique avait gagné une nouvelle proximité affective et sensible avec les vivants.

\author{
Fabienne Duteil-Ogata \\ UMR 8177, EHESS, Paris \\ CLARE EA 4593 \\ Université Bordeaux Montaigne
}

fabienne.duteil-ogata@u-bordeaux-montaigne.fr

\begin{abstract}
Résumé
Cet article propose d'examiner les lieux de sépulture et lieux cultuels destinés aux morts au Japon à partir des pratiques funéraires contemporaines afin de considérer les changements survenus depuis le début du XXI' siècle et poser la question d'une nouvelle ontologie du mort. S'appuyant sur plusieurs terrains ethnographiques réalisés en milieu urbain, principalement à Tôkyô, l'étude des nouvelles pratiques montre que l'espace social de la lignée ancestrale tend à s'élargir à des personnes sans lien de parenté, voire même aux animaux de compagnie alors que l'espace de la sépulture et l'espace cultuel, au contraire, tendent à prendre moins d'espace. En effet, les tombes traditionnellement liées au groupe domestique deviennent des tombes collectives, les cendres des morts sont dispersées dans la nature réduisant le défunt à un élément naturel ou bien encore les objets cinéraires (contenant des cendres ou fabriqués à base de cendres), concentrent dans un artefact portable et mobile le défunt. Assiste-t-on à un changement de paradigme, à une nouvelle ontologie du mort au Japon ?
\end{abstract}

\title{
Mots-clés
}

Japon, pratiques funéraires, mort, tombe, lancer des cendres, objet cinéraire, culte des ancêtres. 


\section{Abstract}

This article proposes to examine the burial places and places of worship intended for the dead in Japan from contemporary funeral practices in order to consider the changes that have occurred since the beginning of the $21^{\text {st }}$ century and raise the question of a new ontology of the dead. Drawing on several ethnographic fieldworks carried out in an urban environment, mainly in Tokyo, the study of new practices shows that the social space of the ancestral lineage tends to widen to people unrelated, even to pet while the burial space and the cult space, on the contrary tend to take up less space. Indeed, the graves traditionally linked to the domestic group become collective graves, the ashes of the dead are dispersed in nature reducing the deceased to a natural element or even cinerary objects (containing ashes or made from ash), concentrate in a portable and mobile artifact the deceased. Are we witnessing a paradigm shift to a new ontology of the dead in Japan?

\section{Keywords}

Japan, Burial Rituals, death, burial spaces, Ash scattering, cinerary objects, Ancestor worship. 